\title{
New Aspects of Pathogenesis and Treatment of Membranous Glomerulopathy After the MENTOR Study
}

The role of podocytes in membranous nephropathy ( $M N$ ) has been recognised for several years and has led to innovations in treatment of $\mathrm{MN}$, with drugs increasingly becoming more specific and efficacious. This timely review evaluates the latest clinical findings regarding the pathogenesis and treatment of $\mathrm{MN}$, from supportive to immunosuppressive EDITOR'S therapies. With 10,000 new cases a year in Europe, optimising the treatment pathway for patients with $\mathrm{MN}$ is imperative.

Authors:

Disclosure:

Received:

Accepted:

Keywords:

Citation:
*Maurizio Salvadori, ${ }^{1}$ Aris Tsalouchos ${ }^{2}$

1. Department of Transplantation, Careggi University Hospital, Florence, Italy

2. Division of Nephrology, Saints Cosmas and Damian Hospital, Pescia, Italy

*Correspondence to maurizio.salvadori1@gmail.com

The authors have declared no conflicts of interest.

The authors would like to thank Dr Stefanos Tsaloucos for their help with figure drawing.

01.03 .20

13.05 .20

Membranous nephropathy (MN), MENTOR study, new trials, podocyte, rituximab. EMJ Nephrol. 2020;8[1]:46-53.

\section{Abstract}

Membranous nephropathy (MN) is the major cause of nephrotic syndrome in adults, accounting for $20 \%$ of cases with an annual incidence of 1 per 100,000 population. In the past 10 years, the role of podocytes has been identified. Environmental triggers in genetically predisposed patients can activate podocytes to exhibit antigenic epitopes, including PLA2R, THBS1, and NELL1, which become targets of specific autoantibodies with subsequent complement activation. The discovery of these mechanisms has opened a new horizon in the treatment of $M N$, and novel drugs are available with more specific mechanisms of action. Rituximab, a monoclonal antibody directed against CD20 expressed on B lymphocytes, has been used in several trials and appears to induce remission of nephrotic syndrome in $60 \%$ of patients (GEMRITUX trial). The recently published results of the MENTOR trial documented the superior efficacy of rituximab in patients observed for up to 24 months. In MN, the concept of targeting disease control has introduced novel therapies with specific blocking mechanisms, such as belimumab; nonspecific blocking mechanisms, such as those against adrenocorticotropic hormone; and new therapeutic options, such as ofatumumab, bortezomib, and eculizumab, which have recognised the pathological processes involved in the glomerular diseases. 


\section{INTRODUCTION}

Membranous nephropathy (MN) is the most frequent cause of nephrotic syndrome in adults and older patients. It accounts for $20 \%$ of nephrotic syndromes in adults and its annual incidence is 1 per 100,000 population. Overall in Europe, 10,000 new cases are diagnosed per year. In the last 10 years, the pathogenetic mechanisms have been defined which has opened up new ways of treatment.

\section{RESEARCH METHODOLOGY}

This paper reviews the latest insights into the pathogenesis and treatment of $M N$. The authors analysed available papers on $M N$ pathogenesis and $M N$ therapy by performing a literature search using PubMed with the search terms "MN pathogenesis" and "MN therapy." Firstly, the papers published in the last 3 years were examined. Paper selection was made according to the relevance of the journal, the authors, the dimension of the study, and the novelty of the findings, with 20 recently published papers selected. The authors then looked to older publications, and studies previously published were also included. Currently ongoing studies were searched for in "https://clinicaltrials.gov/" and randomised controlled trials (RCT) that were active and enrolling patients were included. Those that had not started or were closed were not included. Overall, the story of $\mathrm{MN}$ is a very long one and it has not yet finished. This literature review covers from the first studies in 1995, up to the time of writing.

\section{AETIOLOGY AND PATHOGENESIS}

In recent years, $\mathrm{MN}$ has been found to be essentially a disease of the podocyte which, as a response to environmental triggers and on a genetic basis, exhibits antigen epitopes which bind antibodies that are then able to bind complement. The first antigen to be recognised was NEP by Debiec et al. ${ }^{2}$ Later, a different podocyte protein, the M-type PLA2R, was identified as the antigen responsible for $70-80 \%$ of $M N{ }^{3}$ Because PLA2R is a normal molecule of the podocyte structure, $\mathrm{MN}$ may be regarded as an autoimmune disease, at least in those patients in whom anti-PLA2R antibodies may be found. ${ }^{4}$ The discovery of M-type PLA2R as a major antigen in idiopathic $M N$ (iMN) was a breakthrough in the understanding of the pathogenesis of this disease, establishing iMN as an autoimmune disease. Subsequent studies confirmed that circulating antibodies against PLA2R were detected in approximately $70 \%$ of incident iMN patients. Recently, it has been shown that the presence of PLA2R antibodies supported a diagnosis of iMN, changes in antibody levels were related to clinical disease activity, disappearance of antibodies preceded and predicted a subsequent decrease of proteinuria, and high titres of antibodies were associated with a low likelihood of spontaneous remission. ${ }^{5}$ Recently, another podocyte antigen, THSD7A, has been found to be responsible for around $10 \%$ of $M N^{6}$

In a recent study of PLA2R-negative patients with $M N$, the technique of laser dissection of glomeruli followed by mass spectrometry allowed the identification of the presence of the protein NELL-1 that was also localised by immunohistochemistry. ${ }^{7}$ The authors concluded that a subset of $\mathrm{MN}$ is associated with colocalisation of NELL-1 and Ig G along the glomerular basement membrane. In these patients, antiNELL antibodies were also found in the serum. Additionally, the antigens of aldose reductase and superoxide dismutase have been suggested in some cases of $M N .{ }^{8}$ As a consequence of these findings, for a better understanding of the disease and of possible new therapies, studies are now looking for new podocyte markers that are able to activate the complement cascade and for cells that are able to produce the antibodies involved. 9,10

The Toronto Glomerulonephritis Registry determined the Toronto Risk Score, dividing patients into those at low risk for progression, intermediate risk, and high risk, according to their proteinuria levels in the first 6 months." van den Brand et al. ${ }^{12}$ found markers predictive of evolution towards renal failure in a1 and b2 microglobulin excretions. ${ }^{13}$ The best marker of the disease evolution and determinant for treatment is the titre of anti-PLA2R. ${ }^{14-16}$ 


\section{TREATMENT}

\section{Supportive Treatment}

In the first period of the disease, supportive treatment without the use of immunosuppressants is recommended by the Kidney Disease Improving Global Outcomes (KDIGO) guidelines for all patients with $\mathrm{MN}$ and nephrotic syndrome. ${ }^{17}$ Recommended treatment consists of restricting dietary sodium intake to $<2 \mathrm{~g} /$ day and controlling blood pressure, hyperlipidaemia, and oedema. For all patients, angiotensin-converting enzyme inhibitors and angiotensin receptor blockers should be the first-line therapy because of their antiproteinuric effect. $^{18}$ If the nephrotic syndrome persists, or patients have a recurrence of nephrotic syndrome in the first 6 months of treatment, immunosuppressive treatment should be considered.

\section{Immunosuppressive Treatment}

The combination of corticosteroids with cyclophosphamide or chlorambucil, given over 6 months, is best-known as 'Ponticelli's regimen.' Several studies have documented a remission rate of $70-80 \%$ with this treatment. ${ }^{19-22}$ Cyclophosphamide and chlorambucil are equally effective in inducing remission, but one study ${ }^{2}$ has documented a better tolerability profile for cyclophosphamide. A prophylactic treatment with trimethoprim sulfamethoxazole is recommended to avoid pneumocystis pneumonia. ${ }^{23}$

\section{Anti-Calcineurin Drugs}

Prospective, randomised studies have documented the efficacy of cyclosporine $A$ (CSA) and tacrolimus (TAC) in the treatment of $M N{ }^{24-26}$ In addition to their immunosuppressive effect, CSA and TAC have an antiproteinuric effect because of their action on podocyte structure via interaction with synaptopodin. ${ }^{27}$ The main drawbacks of CsA and TAC are their nephrotoxicity and the high rates of recurrence when the drugs are reduced. ${ }^{28}$

\section{Mycophenolate Mofetil}

Several observational studies have suggested that mycophenolate mofetil (MMF) is effective for the treatment of $M N{ }^{29}$ However, the only published controlled study did not document such efficacy. ${ }^{30}$ For this reason, although the combination of MMF with a high dose of corticosteroids appears effective, the KDIGO guidelines do not recommend MMF as the firstline of treatment for patients with $M N{ }^{17}$

\section{Rituximab}

Rituximab (RTX) is a monoclonal antibody directed against CD2O on the surface of $B$ lymphocytes. In the case of MN, RTX is used to block the production of antibodies directed against the antigens that characterise the $\mathrm{MN}$. Previous observational studies documented the efficacy of RTX in MN, with complete or partial remission of the nephrotic syndrome in 60\% of patients. ${ }^{31-34}$ No treatment-related serious adverse events were reported in either study. The RTX doses used in the different studies have principally been $375 \mathrm{mg} / \mathrm{m}^{2} /$ week for 4 weeks, or one or two 19 doses. More recently, a controlled, prospective, randomised trial commenced (GEMRITUX trial), ${ }^{35}$ comparing two doses of $375 \mathrm{mg} / \mathrm{m}^{2}$ with supportive treatment versus supportive treatment alone in 75 patients with MN. The study results at 17 months documented a remission rate for patients treated with RTX of $65 \%$ versus $34 \%$ in supportive treatment alone $(p<0.03)$.

\section{INSIGHTS FROM THE MENTOR STUDY}

The recent publication of the first 24 months of data from the MENTOR study has allowed for better clarification of the role and relevance of RTX in the treatment of iMN. 36,37 The MENTOR study is a landmark investigator-initiated, open-label, randomised, noninferiority trial conducted in North America. In the study, 130 patients with primary $\mathrm{MN}$, almost all positive for PLA2R, were randomised to receive RTX or CsA. The primary endpoint was complete remission or partial remission of proteinuria at 24 months post-randomisation.

Patients assigned to receive RTX were given a dose of $1 \mathrm{~g}$ on Day 1 and on Day 15. If complete remission was attained at 6 months, no additional RTX was given, but if proteinuria decreased by $>25 \%$ from baseline at 6 months, a second course of RTX was given. When proteinuria did not decrease by $>25 \%$ at 6 months, it was considered a treatment failure. Patients assigned to CsA were given $3.5 \mathrm{mg} / \mathrm{kg} /$ day in two equally 
divided doses. If complete remission was attained at 6 months, CsA was stopped. If proteinuria decreased by $>25 \%$ from baseline at 6 months, CsA was continued for an additional 6 months. A treatment failure was considered when proteinuria decreased $<25 \%$ at 6 months. All patients were followed for 24 months.

The cumulative treatment failure for each treatment is shown in Table 1. The difference in favour of RTX is both high and significant, particularly at 24 months. Table 2 shows the response at 24 months according to baseline anti-PLA2R levels, and again documents a significant response in favour of RTX. Overall, the MENTOR study documented that RTX was not inferior to CsA in inducing complete or partial remission of proteinuria and was superior in maintaining proteinuria remission up to 24 months.

\section{WHAT SHOULD BE THE INITIAL THERAPY FOR PRIMARY MEMBRANOUS NEPHROPATHY?}

The first-line, initial therapy should be either RTX or cyclophosphamide (the latter according to Ponticelli's regimen). A cyclophosphamide regimen may be preferred for patients with very high levels of anti-PLA2R, even if the clinical response does not seem to be predicted by PLA2R at baseline. There is currently an Italian RCT underway, the RICYCLO trial, ${ }^{38}$ comparing RTX to cyclophosphamide. CsA is now considered the second-line therapy for MN, with MMF, Acthar ${ }^{\circledR}$ gel, and plasma exchange as third-line therapies.

Table 1: MENTOR trial: cumulative treatment failure.

\begin{tabular}{|l|c|c|c|}
\hline $\begin{array}{l}\text { Time from randomisation } \\
(\text { months })\end{array}$ & $\begin{array}{c}\text { Rituximab } \\
(\mathrm{n}=65)\end{array}$ & $\begin{array}{c}\text { Cyclosporine A } \\
(\mathrm{n}=65)\end{array}$ & Risk difference \\
\hline 6 & $26 \%$ & $29 \%$ & -3.1 \\
\hline 12 & $26 \%$ & $32 \%$ & -6.2 \\
\hline 18 & $27 \%$ & $74 \%$ & -46.0 \\
\hline 24 & $40 \%$ & $80 \%$ & -40.0 \\
\hline
\end{tabular}

Table 2: MENTOR trial: baseline anti-PLA2R levels and response at 24 months.

\begin{tabular}{|l|c|c|}
\hline Anti-PLA2R level at baseline & $\begin{array}{c}\text { Rituximab } \\
\text { (CR/PR) }\end{array}$ & $\begin{array}{c}\text { Cyclosporine A } \\
\text { (CR/PR) }\end{array}$ \\
\hline$\leq 40 \mathrm{U} / \mathrm{mL}$ & $11 / 15=73 \%$ & $7 / 19=37 \%$ \\
\hline$>40 \mathrm{U} / \mathrm{mL}$ & $28 / 50=56 \%$ & $6 / 46=13 \%$ \\
\hline Total & $39 / 65=60 \%(23 \mathrm{CR})$ & $13 / 65=20 \%(\mathrm{O} \mathrm{CR})$ \\
\hline
\end{tabular}

CR: complete remission; PR: partial remission. 


\section{Insights from MENTOR}

- $\quad$ RTX is the preferred initial therapy of apparently primary MN (irrespective of anti-PLA2R levels) compared to CsA, after 24 months of observation.

- RTX is safe and well-tolerated over the short-term.

- A 40\% failure rate at 24 months for RTX and an $80 \%$ failure rate of CsA at 24 months indicates that additional unmet needs exist in the treatment of primary $\mathrm{MN}$, but may have been, in part, because of the trial design.

\section{Unanswered questions from MENTOR}

- What is the influence of spontaneous remissions on results? (no placebo control)

- What is the efficacy and safety of RTX in comparison to CyC-based regimens?

- What is the influence of anti-PLA2R positive/negative status on efficacy? (no stratification for anti-PLA2R levels)

- Is there any clinical value of monitoring CD19/20 B cells in circulation?

- Would more prolonged CSA therapy or more repeat dosing of RTX influence results?

- Will RTX therapy prevent ESRD?

CD: cluster of differentiation; CsA: cyclosporine A; CyC: cyclophosphamide; ESRD: end-stage renal disease; MN: membranous nephropathy; RTX: rituximab.

\section{What Can Be Done to Augment the Response to RTX?}

Higher doses of RTX can be used when anti-PLA2R levels are very high. Combination treatment of RTX with low-dose cyclophosphamide for 3-6 months may be considered, but a RCT is needed. Ofatumumab, obinutuzumab, or other drugs mentioned below may be used to augment the response to RTX but, again, an $\mathrm{RCT}$ is needed. Adjunctive plasma exchange or immunoadsorption ${ }^{39}$ may also be considered, or combination therapy with sequential calcineurin inhibitors, as is undergoing testing in the STARMEN trial. ${ }^{40}$

\section{How do Anti-PLA2R Antibody Levels Influence the Treatment of Primary MN?}

This issue was not tested in the MENTOR study because no placebo control or stratification for anti-PLA2R levels was undertaken. However, from the available data, high PLA2R levels were associated with higher resistance to treatment, while low levels were associated with a high spontaneous remission rate. In addition, declining serum anti-PLA2R levels predicted clinical remission, while rising levels predicted relapse. However, CsA was much less effective in lowering anti-PLA2R antibody levels.

\section{Unanswered Questions}

The efficacy and safety of RTX in comparison to cyclophosphamide-based regimens is unanswered by the MENTOR study, but the RI-CYCLO trial aims to determine this. It is unclear from the MENTOR study what the influence of anti-PLA2R antibody status is on the efficacy of RTX because no stratification for anti-PLA2R levels was made. The study does not clarify if there is any clinical value in monitoring CD19/20 B cells in circulation. Finally, the study does not answer the question as to whether RTX therapy will prevent end-stage renal disease. Box 1 summarises the insights from the MENTOR Study, as well as the unanswered questions.

\section{Ongoing Trials with Rituximab}

There are several ongoing trials comparing RTX with cyclophosphamide or anti-calcineurin drugs, using different RTX doses. There are four ongoing RCT with RTX. The peptide GAM immunoadsorption therapy in autoimmune 
membranous nephropathy (PRISM) ${ }^{41}$ study is based on the understanding that $\mathrm{iMN}$ is an autoimmune disease characterised by the presence of IgG autoantibodies to M-type PLA2R. Immunoadsorption is a method of removing specific circulating immunoglobulins and has been shown to remove $>80 \%$ of circulating IgG with a single session of 2.5 plasma volumes, with albumin and antithrombin III unaffected; with multiple sessions this can rise to $>98 \%$. The sequential treatment with TAC-RTX versus steroids plus cyclophosphamide in patients in the $\mathrm{iMN}(\mathrm{STARMEN})$ trial $^{40}$ will compare a TAC-RTX treatment with Ponticelli's regimentreated groups. Rates of remission, relapse, and preservation of renal function will be evaluated at a 2-year follow-up. ${ }^{42}$ Trial NCTO097797743 compares RTX to CsA. The CsA group will withdraw CsA after 6 months and introduce RTX. The RI-CYCLO trial ${ }^{38}$ is recruiting MN patients in Italy to compare the efficacy of RTX with Ponticelli's regimen.

\section{ADRENOCORTICOTROPIC HORMONE}

Adrenocorticotropic hormone (ACTH) stimulates the production of endogenous glucocorticoids and activates the melanocortin receptors, which perform several functions including immunomodulation, anti-inflammation, and modulation of exocrine functions. ${ }^{44}$ In rodents, these receptors have been found in podocytes and glomerular endothelial, mesangial, and tubular epithelial cells. In animal models affected by $\mathrm{iMN}$, the inhibition of melanocortin receptors reduces proteinuria and improves podocyte morphology. ${ }^{45}$ After a first pilot study, ${ }^{46}$ two studies ${ }^{47,48}$ demonstrated the beneficial effects of natural ACTH in resistant glomerular diseases. Hladunewich et al., ${ }^{49}$ in a prospective open-label study, confirmed these beneficial results in 20 patients affected by iMN. To date, two ongoing studies are registered on clinicaltrials.gov. .0,51 $^{2}$

\section{NEW EXPERIENCES}

\section{Ofatumumab}

Ofatumumab is a new monoclonal antibody acting on CD2O. It differs from RTX because it has different target epitopes. Ofatumumab, in addition to acting on the same epitope as is recognised by RTX, also acts on a second epitope localised on the small loop of CD2O and on a portion of the large, extracellular loop. Ofatumumab has been assessed as a RTX rescue therapy. Ruggenenti et al. ${ }^{52}$ recently described two cases of clinical remission of iMN in patients who developed primary and secondary resistance to RTX. Resistance to RTX in these cases could be as a result of a change in the CD2O antigen conformation, which prevents B-cell-RTX binding and the consequent B-cell depletion.

\section{Belimumab}

As a monoclonal antibody, belimumab specifically targets the soluble form of B-lymphocyte stimulator that has a critical role in the differentiation and homeostasis of $B$ lymphocytes. The effects of belimumab on proteinuria and anti-PLA2R antibody production have been evaluated in 14 patients with antiPLA2R-positive MN. The treatment significantly reduced the antibody titre and proteinuria within 12 weeks. ${ }^{53}$ Changes in proteinuria and in antiPLA2R antibody titre after belimumab treatment seemed to parallel the changes observed after RTX, with a delay in onset. This may reflect the immediate B-cell lysis achieved by RTX, whereas the slower effect of belimumab might reflect the progressive 'exhaustion' of antibody-producing B cells secondary to B-lymphocyte stimulator binding and inhibition.

\section{Targeting Memory Plasma Cells}

The advanced stages of MN could be mediated primarily by autoreactive plasma cells, which are resistant to anti-CD2O monoclonal antibodies but sensitive to anti-CD38 antibodies or proteasome inhibitors. ${ }^{52}$ Memory plasma cells survive RTX because they do not express the CD2O antigen; plasma cells express CD38. ${ }^{54,55}$ These autoreactive plasma cells could be a target for anti-CD38 monoclonal antibodies, such as daratumumab and isatuximab. To date, these agents have been developed to kill malignant plasma cells. ${ }^{55}$ Other molecules, such as the proteasomeinhibitor bortezomib, may effectively deplete plasma cells. Bortezomib acts by causing an intracellular accumulation of abnormal proteins with consequent plasma cell apoptosis. To date, bortezomib has been used in antineutrophil cytoplasmic antibody nephritis ${ }^{56}$ and in resistant systemic lupus erythematosus. ${ }^{57}$ Preliminary 
data suggest it may be useful in the treatment of iMN that is resistant to other therapies. ${ }^{58,59}$ The main drawback of bortezomib is its toxicity, which necessitates treatment interruption in most patients.

\section{Targeting Complement}

Complement inhibition by the anti-C5
monoclonal antibody eculizumab could
be another avenue for treating $\mathrm{iMN}$. In this approach, complement inhibition could prevent glomerular damage that occurs prior to the removal of antibodies. ${ }^{60}$ To date, attempts to treat membranous nephropathy by eculizumab have failed and studies remain unpublished.

\section{CONCLUSION}

The discovery of anti-PLA2R antibodies and other antibodies involved in the pathogenesis of $\mathrm{MN}$ has revolutionised the treatment approach to this disease; for the first time, iMN may be considered an autoimmune disease in which podocytes play the initial and most important role. The possibility of monitoring anti-PLA2R antibodies represents an important tool for nephrologists to monitor the disease and to check the therapeutic effects. Other autoantibodies have been recognised as causative in iMN, including autoantibodies against THSD7A and NELL-1. Few iMN remain to be explained, but it is likely only a short time until other antigens and autoantibodies will be discovered.

After the publication of the first data of the MENTOR trial, RTX represents the most important drug in the treatment of iMN. Several relevant questions remain to be answered. What is the most appropriate dosage? What is the role of other immunosuppressants? What is the role of PLA2R or other autoantibodies at baseline? How should relapses be treated? Several ongoing RCT aim to answer these questions. Additionally, the pharmaceutical pipeline is filled with other new drugs which are all the subject of RCT. Ofatumumab for MN resistant to RTX, drugs targeting the memory plasma cells, and drugs affecting the complement pathway seem to be the most important therapies for future study and potential treatment of $\mathrm{MN}$.

\section{References}

1. Ronco P, Debiec H. Membranous nephropathy: a fairy tale for immunopathologists, nephrologists and patients. Mol Immunol. 2015;68(1):57-62.

2. Debiec $\mathrm{H}$ et al. Antenatal membranous glomerulonephritis due to anti-neutral endopeptidase antibodies. N Engl J Med. 2002;346(26):2053-60.

3. Beck LH Jr et al.M-type phospholipase $A_{2}$ receptor as target antigen in idiopathic membranous nephropathy. N Engl J Med. 2009;361(1):11-21.

4. Stanescu HCet al. Risk HLA-DQA1 and PLA(2)R1 alleles in idiopathic membranous nephropathy. N Engl J Med. 2011;364(7):616-26.

5. Netti GS, Ranieri E.[Antiphospholipase A2 receptor (antiPLA2R) antibodies and idiopathic membranous nephropathy: which role in diagnosis and prognosis of this disease?]. G Ital Nefrol. 2014;31(3). (In Italian).

6. Tomas NM et al. Thrombospondin Type-1 domain-containing 7A in idiopathic membranous nephropathy. N Engl J Med. 2014;371(24):2277-87.
7. Sethi S et al. Neural epidermal growth factor-like 1 protein (NELL-1) associated membranous nephropathy. Kidney Int. 2020;97(1):163-74.

8. Prunotto $M$ et al. Autoimmunity in membranous nephropathy targets aldose reductase and SOD2. J Am Soc Nephrol. 2010;21(3):507-19.

9. Cattran DC, Brenchley PE. Membranous nephropathy: integrating basic science into improved clinical management. Kidney Int. 2017;91(3):566-74.

10. Ronco P, Debiec $\mathrm{H}$. Pathophysiological advances in membranous nephropathy: time for a shift in patient's care. Lancet. 2015;385(9981):1983-92.

11. Cattran DC et al. Validation of a predictive model of idiopathic membranous nephropathy: its clinical and research implications. Kidney Int. 1997;51:901-7.

12. van den Brand JA et al. Lowmolecular-weight proteins as prognostic markers in idiopathic membranous nephropathy. Clin J Am Soc Nephrol. 2011;6(12):2846-53.

13. Branten AJ et al. Urinary excretion of beta2-microglobulin and IgG predict prognosis in idiopathic membranous nephropathy: a validation study. J Am Soc Nephrol. 2005;16(1):169-74.

14. Hofstra JM et al. Anti-phospholipase $\mathrm{A}_{2}$ receptor antibodies correlate with clinical status in idiopathic membranous nephropathy. Clin J Am Soc Nephrol. 2011;6(6):1286-91.

15. Beck LH Jr et al. Rituximabinduced depletion of anti-PLA2R autoantibodies predicts response in membranous nephropathy. J Am Soc Nephrol. 2011;22(8):1543-50.

16. De Vriese AS et al. A proposal for a serology-based approach to membranous nephropathy. J Am Soc Nephrol. 2017;28(2):421-30.

17. Kidney Disease Improving Global Outcomes (KDIGO) glomerulonephritis working group. Chapter 7: idiopathic membranous nephropathy. Kidney Int Suppl (2011). 2012;2(2):186-97.

18. Polanco $\mathrm{N}$ et al. Spontaneous remission of nephrotic syndrome in membranous nephropathy with chronic renal impairment. Nephrol Dial Transplant. 2012;27(1):231-4.

19. Ponticelli $C$ et al. A 10 -year followup of a randomized study with 
methylprednisolone and chlorambuci in membranous nephropathy. Kidney Int. 1995;48(5):1600-4.

20. Ponticelli $\mathrm{C}$ et al. A randomized study comparing methylprednisolone plus chlorambucil versus methylprednisolone plus cyclophosphamide in idiopathic membranous nephropathy. J Am Soc Nephrol. 1998;9(3):444-50.

21. Jha $V$ et al. A randomized, controlled trial of steroids and cyclophosphamide in adults with nephrotic syndrome caused by idiopathic membranous nephropathy J Am Soc Nephrol. 2007;18(6):1899904.

22. Pani A. Standard immunosuppressive therapy of immune-mediated glomerular diseases. Autoimmun Rev. 2013;12(8):848-53.

23. Torres A et al. Conservative versus immunosuppressive treatment of patients with idiopathic membranous nephropathy. Kidney Int. 2002;61(1):219-27.

24. Cattran DC et al. Cyclosporine in patients with steroid-resistant membranous nephropathy: a randomized trial. Kidney Int. 2001;59(4):1484-90.

25. Caro J et al. Predictors of response and relapse in patients with idiopathic membranous nephropathy treated with tacrolimus. Nephrol Dial Transplant. 2015;30(3):467-74.

26. Alexopoulos E et al. Induction and long-term treatment with cyclosporine in membranous nephropathy with the nephrotic syndrome. Nephrol Dial Transplant. 2006;21(11):3127-32

27. Mathieson PW. Proteinuria and immunity--an overstated relationship? N Engl J Med. 2008;359(23):2492-4.

28. Segarra A et al. Successful treatment of membranous glomerulonephritis with rituximab in calcineurin inhibitordependent patients. Clin J Am Soc Nephrol. 2009;4(6):1083-8.

29. Choi MJ et al. Mycophenolate mofetil treatment for primary glomerular diseases. Kidney Int. 2002;61(3):1098114.

30. Dussol B et al. Mycophenolate mofetil monotherapy in membranous nephropathy: a 1-year randomized controlled trial. Am J Kidney Dis. 2008;52(4):699-705.

31. Fervenza FC et al. Rituximab treatment of idiopathic membranous nephropathy. Kidney Int. 2008;73(1):117-25.

32. Fervenza FC et al. Rituximab therapy in idiopathic membranous nephropathy: a 2-year study. Clin J Am Soc Nephrol. 2010;5(12):2188-98.

33. Ruggenenti $P$ et al. Rituximab in idiopathic membranous nephropathy J Am Soc Nephrol. 2012;23(8):141625.

34. Busch $M$ et al. Rituximab for the second-and third-line therapy of idiopathic membranous nephropathy: a prospective single center study using a new treatment strategy. Clin Nephrol. 2013;80(2):105-13.

35. Dahan $\mathrm{K}$ et al. Rituximab for severe membranous nephropathy: a 6-month trial with extended follow-up. J Am Soc Nephrol. 2017;28(1):348-58.

36. Mayo Clinic. Membranous Nephropathy Trial Of Rituximab (MENTOR). NCT01180036. https:// clinicaltrials.gov/ct2/show/ NCT01180036.

37. Fervenza FC et al. Rituximab or cyclosporine in the treatment of membranous nephropathy. N Engl J Med. 2019;381(1):36-46.

38. Azienda Socio Sanitaria Territorialedegli Spedali Civili di Brescia. rituximab versus steroids and cyclophosphamide in the treatment of idiopathic membranous nephropathy (RI-CYCLO). NCT03018535. https://clinicaltrials. gov/ct2/show/NCT03018535.

39. Kronbichler $\mathrm{A}$ et al. Immunoadsorption in nephrotic syndrome: where are we now and where are we going from here? Atheroscler Suppl. 2019;40:55-60.

40. Hospital Universitario 12 de Octubre. Sequential Therapy With Tacrolimus and Rituximab in Primary Membranous Nephropathy (STARMEN). NCTO1955187. https:// clinicaltrials.gov/ct2/show/ NCT01955187.

41. Manchester University NHS Foundation Trust. Peptide GAM Immunoadsorption Therapy in Autoimmune Membranous Nephropathy (PRISM). NCTO3255447. https://clinicaltrials.gov/ct2/show/ NCTO3255447.

42. Rojas-Rivera J al. A European multicentre and open-label controlled randomized trial to evaluate the efficacy of Sequential treatment with TAcrolimus-Rituximab versus steroids plus cyclophosphamide in patients with primary MEmbranous Nephropathy: the STARMEN study. Clin Kidney J. 2015;8(5):503-10.

43. National Institute of Diabetes and Digestive and Kidney Diseases (NIDDK). Rituximab Plus Cyclosporine in Idiopathic Membranous Nephropathy. NCTO0977977. https://clinicaltrials. gov/ct2/show/NCT00977977.

44. Gong R. The renaissance of corticotropin therapy in proteinuric nephropathies. Nat Rev Nephrol. 2011;8(2):122-8.

45. Lindskog A et al. Melanocortin 1 receptor agonists reduce proteinuria. J Am Soc Nephrol. 2010;21(8):1290-8.

46. Berg $A L$ et al. Beneficial effects of $\mathrm{ACTH}$ on the serum lipoprotein profile and glomerular function in patients with membranous nephropathy. Kidney Int. 1999;56(4):1534-43.

47. Ponticelli $\mathrm{C}$ et al. A randomized pilot trial comparing methylprednisolone plus a cytotoxic agent versus synthetic adrenocorticotropic hormone in idiopathic membranous nephropathy. Am J Kidney Dis. 2006;47(2):233-40.

48. Bomback AS et al. Treatment of resistant glomerular diseases with adrenocorticotropic hormone gel: a prospective trial. Am J Nephrol. 2012;36(1):58-67.

49. Hladunewich MA et al. A pilot study to determine the dose and effectiveness of adrenocorticotrophic hormone (H.P. Acthar ${ }^{\circledR} \mathrm{Gel}$ ) in nephrotic syndrome due to idiopathic membranous nephropathy. Nephrol Dial Transplant. 2014;29(8):1570-7.

50. Radboud University. Treatment With Synthetic ACTH in High Risk Patients With Membranous Nephropathy (ACTHiMeN). NCT00694863. https://clinicaltrials.gov/ct2/show/ NCT00694863.

51. Icahn School of Medicine at Mount Sinai. Adrenocorticotropic Hormone in Membranous Nephropathy. NCT03025828. https://clinicaltrials. gov/ct2/show/NCTO3025828.

52. Ruggenenti $P$ et al. Treatment of membranous nephropathy: time for a paradigm shift. Nat Rev Nephrol. 2017;13(9):563-79.

53. Willcocks $L$ et al. Effect of belimumab on proteinuria and ant PLA2R autoantibody in idiopathic membranous nephropathy - 6 months data. Abstract $\mathrm{SaO0} 22$. ERA-EDTA Congress, 28-31 May, 2015.

54. Mei HE et al. A unique population of lgG-expressing plasma cells lacking CD19 is enriched in human bone marrow. Blood. 2015;125(11):1739-48.

55. van de Donk NW et al.Monoclonal antibodies targeting CD38 in hematological malignancies and beyond. Immunol Rev. 2016;270(1):95112.

56. Novikov Pet al. Bortezomib in refractory ANCA-associated vasculitis: a new option? Ann Rheum Dis. 2016;75(1):e9.

57. Alexander Tet al. The proteasome inhibitior bortezomib depletes plasma cells and ameliorates clinical manifestations of refractory systemic lupus erythematosus. Ann Rheum Dis. 2015:74(7):1474-8.

58. Barbari A et al. Bortezomib as a novel approach to early recurrent membranous glomerulonephritis after kidney transplant refractory to combined conventional rituximab therapy. Exp Clin Transplant. 2017;15(3):350-4

59. Hartono $\mathrm{C}$ et al. Bortezomib therapy for nephrotic syndrome due to idiopathic membranous nephropathy. J Nephrol. 2014;27(1):103-6.

60. Borza DB. Alternative pathway dysregulation and the conundrum of complement activation by IgG4 immune complexes in membranous nephropathy. Front Immunol. 2016;7:157. 